\title{
A Rare Case of Resolution of High-Degree Atrioventricular Block Associated With COVID-19
}

\author{
Farla Jean-Louis ${ }^{\mathrm{a}, \mathrm{c}}$, Ajibola Monsur Adedayo ${ }^{\mathrm{b}}$, Temitope Ajibawo ${ }^{\mathrm{a}}$, \\ Gautham Upadhya ${ }^{\mathrm{b}}$, Asma Syed ${ }^{\mathrm{a}}$
}

\begin{abstract}
Cardiac arrhythmias have been reported in the setting of coronavirus disease 2019 (COVID-19) infection. To date various tachyarrhythmias have been noted. This case report describes an atypical clinical hospitalization course of high-degree atrioventricular (AV) block in the setting of COVID-19 infection which required no intervention. This case highlights the importance of an initial electrocardiogram (ECG) upon presentation and consideration for constant cardiac monitoring in select patients.
\end{abstract}

Keywords: COVID-19; High degree; Atrioventricular; Block; Heart; AV

\section{Introduction}

Coronavirus disease 2019 (COVID-19) is identified as "severe acute respiratory syndrome coronavirus 2" (SARS-CoV-2). Its clinical presentation often included viral upper respiratory symptoms such as fever, cough, shortness of breath and body aches. Cardiac tachyarrhythmias have been commonly reported in association with COVID-19 infection, with sinus tachycardia being the most common type [1]. The most likely pathologic arrhythmias include atrial fibrillation, atrial flutter and monomorphic or polymorphic ventricular tachycardia (VT) [1]. We present an unusual case of transient high-degree atrioventricular block (AVB) in a patient with COVID-19 infection.

\section{Case Report}

We present a 60-year-old Hispanic man with a past medical history (PMHx) significant for hypertension (HTN), non-

Manuscript submitted June 9, 2020, accepted June 19, 2020

Published online July 21, 2020

aBrookdale University Hospital and Medical Center, Brooklyn, NY, USA bSUNY Downstate Medical Center, Brooklyn, NY, USA

${ }^{c}$ Corresponding Author: Farla Jean-Louis, Brookdale University Hospital and Medical Center, Brooklyn, NY, USA. Email: fjean@bhmcny.org

doi: https://doi.org/10.14740/jmc3524 ischemic dilated cardiomyopathy with an ejection fraction of $25 \%$, chronic obstructive pulmonary disease (COPD), hyperlipidemia and marijuana/cocaine abuse (last use was 3 days before admission). The patient was non-compliant with his congestive heart failure (CHF) medications and was not taking any atrioventricular (AV) nodal blocking drugs at the time. He presented to the hospital with complaints of shortness of breath, non-productive cough, subjective fevers, headaches and generalized fatigue. He also reported diarrhea with intermittent abdominal pain but no nausea, vomiting, sick contacts or any recent travel.

Upon presentation, his vital signs were significant for an elevated blood pressure of 192/138 mm Hg, a heart rate of 55 beats per minute (bpm) and an oxygen saturation of $100 \%$ on 2 $\mathrm{L}$ of oxygen via nasal cannula. He was noted to have increased jugular venous distension (JVD), bibasilar lung crackles and an S3 gallop on auscultation. He was found to have a highgrade AV block. The remainder of the physical examination was unremarkable.

An initial 12-lead electrocardiogram (ECG) was obtained on admission, which revealed a high-degree AV block with a right bundle branch block (RBBB) escape morphology (Fig. 1). The patient's previous ECG, in October 2019, demonstrated normal sinus rhythm (NSR) with a left bundle branch block (LBBB) pattern. Urine toxicology screen was positive for cocaine and cannabinoids. A nasopharyngeal swab for SARS$\mathrm{CoV}-2$ virus was positive. The rapid antigen influenza $\mathrm{A}$ and $\mathrm{B}$ swab was negative. His laboratory tests upon admission were significant for thrombocytosis $\left(442 \times 10^{3} / \mu \mathrm{L}\right)$, hyperkalemia $(5.3 \mathrm{mEq} / \mathrm{L})$, elevated liver enzymes (aspartate aminotransferase/alanine aminotransferase (AST/ALT) of 744/601 U/L, respectively), an elevated lactate dehydrogenase (LDH) (1,969 IU/L), elevated D-dimer $(907 \mathrm{ng} / \mathrm{mL})$ (normal $<500 \mathrm{ng} / \mathrm{mL}$ ), lactate $(3.90 \mathrm{mmol} / \mathrm{L})$ and C-reactive protein (CRP) $(3.60 \mathrm{mg} /$ $\mathrm{dL}$ ). Initial troponin I was negative and pro-B-type natriuretic peptide (pro-BNP) was elevated $(11,400 \mathrm{pg} / \mathrm{mL})$. His chest Xray (CXR) showed bilateral pneumonia greater on the left side with cardiomegaly.

He was admitted to a monitored setting and treated for COVID-19 pneumonia without any QTc-prolonging drugs. On day 2 of hospitalization, his ECG had converted to NSR with LBBB, which was consistent with his ECG prior to this admission (Fig. 2). He remained without any recurrence of AV block, had complete resolution of the transient high-degree AVB and remained in NSR with 1:1 AV conduction. He was subsequently discharged home. To date he has not had any worsening 


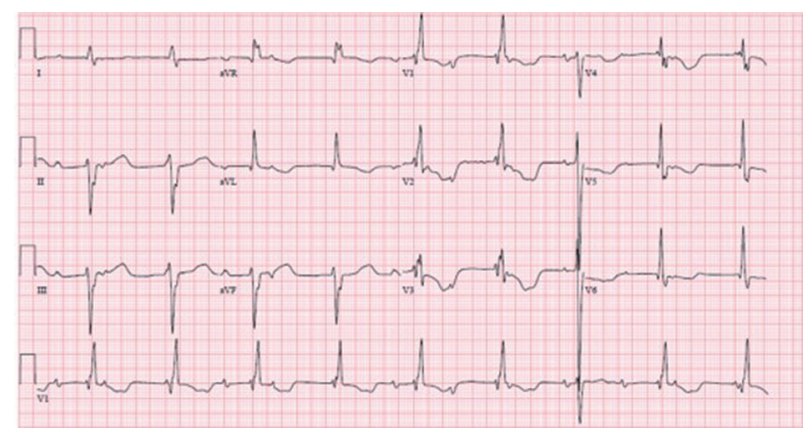

Figure 1. ECG on $3 / 31 / 2020$ shows a high-degree AV block with RBBB left anterior fascicular block, and a fusion complex. HR $54 \mathrm{bpm}$, QRs $156 \mathrm{~ms}$ and QTc $629 \mathrm{~ms}$. ECG: electrocardiogram; AV: atrioventricular; RBBB: right bundle branch block; bpm: beats per minute; HR: heart rate.

bradyarrhythmias or recurrence of AV block.

\section{Discussion}

Bradyarrhythmias such as sinus pauses or high-grade blocks with slow escape rhythms have not been widely reported. The episodes of AV block reported have usually been in the setting of inferior wall myocardial infarctions and/or hypoxia. A significant proportion of COVID-19 patients have cardiovascular disease (CVD) which has been shown to increase its severity and worsened its clinical outcomes. A large report from the Chinese Center for Disease Control and Prevention involving 44,672 confirmed cases of COVID-19 reported a fatality rate of $10.5 \%$ in patients who had CVD compared to an overall $2.3 \%$ fatality rate [2]. SARS-COV-2 affects the human cells, including the cardiovascular system, by binding to the angiotensin-converting enzyme 2 (ACE2) receptors via different mechanisms which can cause myocardial injury [3]. These mechanisms include cytokine storms, myocarditis $[4,5]$ and decreased ACE2 signal pathways as shown in animal studies [3]. Similar pathways may explain the arrhythmogenic effect of COVID-19.

The prevalence of cardiac arrhythmias and cardiac conduction system disease was found to vary from population to population. A study conducted by Fang et al involved 137 subjects, of which only 10 patients described having palpitations [6]. Another study conducted by Wang et al included 138 patients hospitalized for COVID-19-related pneumonia and reported a higher prevalence, with $17 \%$ experiencing arrhythmias from the general cohort, and in $44 \%$ of those admitted to the intensive care unit (ICU) [7]. However, significant electrolyte abnormalities and hypoxemia were also reported in these two studies.

In regards to our case report, two similar cases were identified. The first is of a 54-year-old man with COVID-19 infection which was complicated by acute respiratory failure (requiring intubation) and complete heart block, who needed cardiopulmonary resuscitation (CPR) before spontaneous reversion to NSR [8]. However, the patient was severely hypoxic at the time of the witnessed arrhythmia. The second case was a 66-year-old woman diagnosed with COVID-19 who required

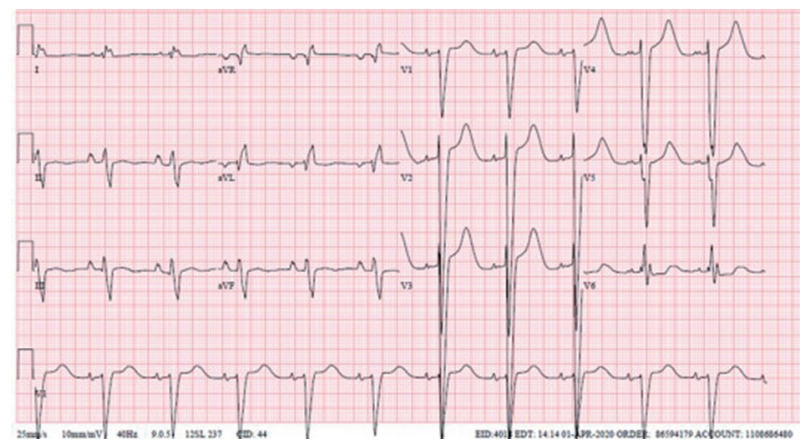

Figure 2. ECG on 4/1/2020 shows NSR, right atrial enlargement, left axis deviation and LBBB. HR $65 \mathrm{bpm}, \mathrm{QRs} 164 \mathrm{~ms}$ and QTc $611 \mathrm{~ms}$. ECG: electrocardiogram; NSR: normal sinus rhythm; LBBB: left bundle branch block; bpm: beats per minute; HR: heart rate.

tracheal intubation and veno-venous extracorporeal membrane oxygenation (VV-ECMO) and experienced a Mobitz type I second-degree AVB, AV junctional escape beat and transient complete AV block which resolved [9]. Hypoxemia may be the cause of the transient complete $\mathrm{AV}$ block in this case as well. Our case differs from these other reports, in that our patient was not hypoxemic nor did he have significant electrolyte abnormalities during the hospitalization while having highdegree AVB.

There are documented causes for COVID-19-related arrhythmias including viral myocarditis, myocardial ischemia, and septic/cardiogenic shock. There also have been case reports of Brugada syndrome being unmasked by fever in a COVID-19-positive patient [10]. Given the increased risk of arrhythmogenic effects related to COVID-19, patients should have an ECG on admission and if there is a high suspicion of further arrhythmias, patients should be monitored on telemetry during hospital course.

\section{Conclusions}

COVID-19 was initially viewed as a predominantly respiratory illness, its pathological effects now known to encompass multiple different organ systems. This case illustrates an unusual finding of AVB that resolved. To date, we have seen COVID-19 patients presenting with inferior wall myocardial infarctions and complete heart block. We have also seen more atrial tachyarrhythmias, but this was a rare case of transient worsening conduction disease without the presence of any other reversible causes.

\section{Acknowledgments}

None to declare.

\section{Financial Disclosure}

None to declare. 


\section{Conflict of Interest}

None to declare.

\section{Informed Consent}

Not applicable.

\section{Author Contributions}

The corresponding author, FJL, wrote the main manuscript. AS provided the idea for the case report, contributed in editing the article and provided the ECG (Figs. 1, 2). AMA provided additional data for the discussion section. TA and GU contributed in editing and formatting the article for submission. All authors reviewed the final manuscript.

\section{Data Availability}

The authors declare that data supporting the findings of this study are available within the article.

\section{References}

1. Jordan MP. Coronavirus disease 2019 (COVID-19): arrhythmias and conduction system disease. (n.d.). 2020. Retrieved from https://www.uptodate.com/contents/coronavirus-disease-2019-covid-19-arrhythmias-and-conduc- tion-system-disease.

2. $\mathrm{Wu} \mathrm{Z}, \mathrm{McGoogan}$ JM. Characteristics of and important lessons from the coronavirus disease 2019 (COVID-19) outbreak in China: summary of a report of 72314 cases from the Chinese center for disease control and prevention. JAMA. 2020.

3. Kuba K, Imai Y, Rao S, Gao H, Guo F, Guan B, Huan $\mathrm{Y}$, et al. A crucial role of angiotensin converting enzyme 2 (ACE2) in SARS coronavirus-induced lung injury. Nat Med. 2005;11(8):875-879.

4. Hu H, Ma F, Wei X, Fang Y. Coronavirus fulminant myocarditis saved with glucocorticoid and human immunoglobulin. Eur Heart J. 2020.

5. Zheng YY, Ma YT, Zhang JY, Xie X. COVID-19 and the cardiovascular system. Nat Rev Cardiol. 2020;17(5):259-260.

6. Liu K, Fang YY, Deng Y, Liu W, Wang MF, Ma JP, Xiao W, et al. Clinical characteristics of novel coronavirus cases in tertiary hospitals in Hubei Province. Chin Med J (Engl). 2020;133(9):1025-1031.

7. Wang D, Hu B, Hu C, Zhu F, Liu X, Zhang J, Wang B, et al. Clinical characteristics of 138 hospitalized patients with 2019 novel coronavirus-infected pneumonia in Wuhan, China. JAMA. 2020.

8. Azarkish M, Laleh Far V, Eslami M, Mollazadeh R. Transient complete heart block in a patient with critical COVID-19. Eur Heart J. 2020;41(22):2131.

9. He J, Wu B, Chen Y, Tang J, Liu Q, Zhou S, Chen C, et al. Characteristic electrocardiographic manifestations in patients with COVID-19. Can J Cardiol. 2020;36(6):966 e961-966 e964.

10. Chang D, Saleh M, Garcia-Bengo Y, Choi E, Epstein L, Willner J. COVID-19 infection unmasking Brugada syndrome. HeartRhythm Case Rep. 2020. 